\title{
A New Construction of Spheres Via Soft Real Numbers and Soft Points
}

\section{Güzide Şenel}

Department of Mathematics, Amasya University, Amasya, Turkey

\section{Email address:}

g.senel@amasya.edu.tr

\section{To cite this article:}

Güzide Şenel. A New Construction of Spheres Via Soft Real Numbers and Soft Points. Mathematics Letters. Vol. 4, No. 3, 2018 , pp. $39-43$. doi: $10.11648 / \mathrm{j} . \mathrm{ml} .20180403 .11$

Received: August 23, 2018; Accepted: September 19, 2018; Published: October 12, 2018

\begin{abstract}
This study is intended as an attempt to bring together the areas of spheres, soft real numbers and soft points. Relating spheres to soft real numbers and soft points provides a natural and intrinsic construction of soft spheres. In this paper a new construction of spheres is provided via soft real numbers and soft points. This new construction sheds light on soft sphere applications for analyzing the locus of them. Also, several related results have been obtained. It is proved that spheres play an important role in the theory of soft metric spaces with taking into consideration soft points. This viewpoint sheds some new light on soft sphere examples and drawings for analyzing the locus of them. This new approach may be the starting point for soft mathematical concepts and structures based on soft set-theoric operations in soft metric spaces and stimulate the reader to further research.
\end{abstract}

Keywords: Sphere, Soft Real Number, Soft Point, Soft Metric

\section{Introduction}

The inadequacy of the parametrization tool of the classical theories give some difficulties to solve complicated problems in economics, engineering and environment. To overcome these difficulties, Molodtsov introduced the concept of soft set as a new mathematical tool for dealing with uncertainties [1]. After this innovation, soft set theory is applied in various theories.

Some new operations in soft set theory was defined in [2]. The idea of soft matrix theory and its decision making was first studied in [3]. S. Das and S.K. Samanta introduced the concept of soft point and using this concept they have given some new definitions in soft set theory [4]. Every soft point that changes with each parameter that takes place in a soft set was proved in [5]. Soft point's soft matrix form was defined and detailed examination in application of soft set theory was represented in [5]. A possibly more tractable task is to describe soft real sets and soft real numbers was firstly executed by Das and Samanta in [6]. Some topological structures such as soft open sets, soft closed sets and soft closures of soft sets etc. were defined and their properties were studied in [4], synchronously. Soft complex sets and soft complex numbers are defined in [7]. Metric Spaces in pure and applied mathematics is a new area of study in works of $[8,9]$. It is natural to define soft metric spaces by soft sets. The notion of soft metric space was studied, following to this work [4].

Spheres play an important role in the theory of soft metric spaces which is proved in this study with taking into consideration soft points. In this paper a new form of sphere is introduced that is motivated by bring together the areas of spheres, soft real numbers and soft points. This new construction has been shown that a sphere can be reformed by soft real numbers and soft points named as "soft sphere".

\section{Methods}

In the literature, similar studies in soft set theory have been done by several authors. Some of these are as follows: D. Molodtsov, N. Cagman and S. Enginoglu have established important notions of the theory of soft sets $[1,3]$. In Senel (2017), the author has shown that the parameterization reduction of soft point and its applications with soft matrix. Soft positive and negative real numbers have defined in the study of S. Das and S.K. Samanta (2012). In the method of this study, detailed theorems and examples remain loyal to the works of [4]. 


\section{Preliminaries}

In this section, I recall some basic notions of soft set theory which may be found in $[2,10,3,1,11]$ for further details. For a deeper discussion of soft point, I refer the reader to [5]. Also, for detailed definitions, propositions and theorems about soft positive and negative real numbers, the reference [6] can be examined. This case has been thoroughly studied; see [4] for more details, theorems and examples.

Throughout this work, $U$ refers to an initial universe, $E$ is a set of parameters, $A E$ and $P(U)$ is the power set of $U$.

Definition 2.1. ([3,1]) A soft set $f_{A}$ on the universe $\mathrm{U}$ is a set defined by

$$
f_{A}: E \rightarrow P(U) \text { such that } f_{A}(x)=\emptyset \text { if } x \notin A .
$$

Here $f_{A}$ is also called an approximate function. A soft set over $U$ can be represented by the set of ordered pairs

$$
f_{A}=\left\{\left(\mathrm{x}, \mathrm{f}_{\mathrm{A}}(\mathrm{x})\right): \mathrm{x} \in \mathrm{E}, \mathrm{f}_{\mathrm{A}}(\mathrm{x}) \in P(U)\right\} .
$$

Note that the set of all soft sets over $U$ will be denoted by $\mathbb{S}$.

Definition 2.2. ([3]) Let $f_{A} \in \mathbb{S}$. Then,

If $f_{A}(x)=\varnothing$ for all $x \in E$, then $f_{A}$ is called an empty soft set, denoted by $\Phi$.

If $f_{A}(x)=U$ for all $x \in E$, then $f_{A}$ is called universal soft set, denoted by $\tilde{E}$.

If $f_{A}(x)$ is a singleton set for all $x \in E$, then $f_{A}$ is called a singleton soft set.

Definition 2.3. ([6]) Let $X$ be a non-empty set and $E$ be a non-empty parameter set. Then a function $\varepsilon: E \rightarrow \mathrm{X}$ is said to be a soft element of $\mathrm{X}$. A soft element $\varepsilon$ of $\mathrm{X}$ is said to belong to a soft set $\mathrm{A}$ of $\mathrm{X}$, denoted by $\varepsilon \in \mathrm{A}$, if $\varepsilon(\mathrm{e}) \in \mathrm{A}(\mathrm{e}), \forall \mathrm{e} \in E$. Thus a soft set $\mathrm{A}$ of $\mathrm{X}$ with respect to the index set $\mathrm{E}$ can be expressed as $A(\mathrm{e})=\{\varepsilon(\mathrm{e}), \varepsilon \in \mathrm{A}\}, \mathrm{e} \in \mathrm{E}$.

This chapter continues to constitute sufficient preparation by giving definitions of soft real number and soft point:

Definition 2.4. ([6]) Let $\mathbb{R}$ be the set of real numbers, $\rho(\mathbb{R})$ be the collection of all non-empty bounded subsets of $\mathbb{R}, E$ be a set of parameters and $\mathrm{A} \subseteq \mathrm{E}$. Then a mapping $f: A \rightarrow \rho(\mathbb{R})$ is called a soft real set. It is denoted by $(f, A)$. If in particular $(f, A)$ is a singleton soft set, then identifying $(f, A)$ with the corresponding soft element, it will be called a soft real number.

Now on, the single parameter set to be used is denoted by $\mathrm{E}$ to define soft sets and their operations. Thus, the subscript $E$ can be deleted from the soft sets $(f, \mathrm{E})$, i.e, a soft set $f_{\mathrm{E}}$ will be denoted shortly by $\mathrm{f}$ unless this will cause confusion. In general, soft sets and their approximate functions are denoted by $f, g, h, \ldots$, and soft sets and their approximate functions are used interchangeably.
Definition 2.5. ([6]) Let us denote the collection of all soft points of $f$ by $\operatorname{SP}(f)$, the set of all soft real sets by $\mathrm{R}(\mathrm{E})$, the set of all soft real numbers by $\tilde{R}(\mathrm{E})$ and the set of all non-negative soft real numbers by $\tilde{R} *(\mathrm{E})$. If a soft real set is a singleton soft set, it will be called a soft real number and denoted by $\tilde{\mathrm{r}}, \tilde{\mathrm{s}}$ etc.

The best general reference about arithmetic operations of soft real sets and soft real numbers is [4] for a fuller treatment.

Definition 2.6. ([10]) The soft set $f$ is called a soft point in $\mathbb{S}$, if for the parameter $e_{i} \in E$ such that $f\left(e_{i}\right) \neq \varnothing$ and $f\left(e_{j}\right) \neq \varnothing$, for all $e_{j} \in E \backslash\left\{e_{i}\right\}$ is denoted by $\left(\mathrm{e}_{\mathrm{i}_{\mathrm{f}}}\right)_{\mathrm{j}}$ for all $\mathrm{i}, \mathrm{j} \in \mathbb{N}^{+}$. Note that the set of all soft points of $f$ will be denoted by SP $(\mathrm{f})$.

Definition 2.7. ([5]) Let $f \in \mathbb{S}$. Then,

If $f(e)=\varnothing$ for all $\mathrm{e} \in \mathrm{E}$, then $f$ is called null soft point, denoted by $\mathrm{e}_{\Phi}$.

If $f(e)=U$ for all $\mathrm{e} \in \mathrm{E}$, then $f$ is called universal soft point, denoted by $\mathrm{e}_{\tilde{\mathrm{E}}}$.

If there is only one parameter $\mathrm{e} \in \mathrm{E}$ in $f$, then $f$ is denoted by $\mathrm{e}_{\mathrm{f}_{\mathrm{i}}}$. If there is only one parameter $\mathrm{e} \in \mathrm{E}$ in $f$, and $f(\mathrm{e})=\{u\}$ then $f$ is denoted by $\mathrm{e}_{f}$.

Definition 2.8. ([5]) The soft point $\left(\mathrm{e}_{\mathrm{i}_{\mathrm{f}}}\right)_{\mathrm{j}}$ is said to belong to a soft set $g \in \mathbb{S}$, denoted by $\left(\mathrm{e}_{\mathrm{i}_{\mathrm{f}}}\right)_{\mathrm{j}} \notin \mathrm{g}$, if for for the parameter $\mathrm{e}_{\mathrm{i}} \in \mathrm{E}$ and $\mathrm{f}\left(\mathrm{e}_{\mathrm{i}}\right) \subseteq \mathrm{g}\left(\mathrm{e}_{\mathrm{i}}\right)$.

Proposition 1. ([5])

(1) $\mathrm{f} \subseteq g$, if $\left(\mathrm{e}_{\mathrm{i}_{\mathrm{f}}}\right)_{\mathrm{j}} \tilde{\in \mathrm{f}}$ then $\left(\mathrm{e}_{\mathrm{i}_{\mathrm{f}}}\right)_{\mathrm{j}} \tilde{\in} \mathrm{g}$ for all $\mathrm{e}_{\mathrm{i}} \tilde{\in \mathrm{f}}$.

(2) $\mathrm{f}=\mathrm{g}$ if and only if $\left(\mathrm{e}_{\mathrm{i}_{\mathrm{f}}}\right)_{\mathrm{j}} \tilde{\in \mathrm{f}}$ then $\left(\mathrm{e}_{\mathrm{i}_{\mathrm{f}}}\right)_{\mathrm{j}} \tilde{\in} \mathrm{g}$ and $\left(\mathrm{e}_{\mathrm{i}_{\mathrm{f}}}\right)_{\mathrm{j}} \tilde{\epsilon} \mathrm{g}$ and $\left(\mathrm{e}_{\mathrm{i}_{\mathrm{f}}}\right)_{\mathrm{j}} \tilde{\epsilon} \mathrm{f}$ for all $\mathrm{e}_{\mathrm{i}} \tilde{\epsilon} \mathrm{f}$.

A New Construction of Spheres Via Soft Real Numbers and Soft Points

In this a section, an interesting formula for sphere construction is derived with soft real numbers and soft points. This section is intended as an attempt to motivate to bring together the areas in which soft spheres, soft real numbers and soft points.

Definition 3.1. [11] Let $\varnothing \neq \mathrm{X} \subseteq \mathrm{E}, \mathrm{f} \in \mathrm{Sx}(\mathrm{U})$, and $\mathrm{f}: \mathrm{X} \rightarrow \mathrm{P}(U)$ be one to one function. We denote by $\tilde{\mathrm{R}}^{*}(\mathrm{E})$ the set of all soft real numbers and $\mathrm{f}_{\mathrm{i}}, \mathrm{f}_{\mathrm{j}}, \mathrm{f}_{\mathrm{s}} \in \mathrm{S}_{\mathrm{x}}(\mathrm{U})$; $\left(\mathrm{e}_{\mathrm{i}_{\mathrm{f}}}\right)_{\mathrm{j}},\left(\mathrm{e}_{\mathrm{x}_{\mathrm{f}}}\right)_{\mathrm{y}},\left(\mathrm{e}_{\mathrm{s}_{\mathrm{f}}}\right)_{\mathrm{k}} \tilde{\in \mathrm{f}}$. A mapping is said to be a soft metric on the soft set $\mathrm{f}$ if $\tilde{\mathrm{d}}$ satisfies the following conditions: 


$$
\begin{gathered}
d: E ? E \rightarrow \tilde{R} *(E) \\
\left(\left(e_{i_{f}}\right)_{j},\left(e_{x_{f}}\right)_{y}\right) \rightarrow \tilde{d}\left(\left(e_{i_{f}}\right)_{j},\left(e_{x_{f}}\right)_{y}\right) \\
\tilde{d}\left(\left(e_{i_{f}}\right)_{j},\left(e_{x_{f}}\right)_{y}\right) \geq 0 . \\
\tilde{d}\left(\left(e_{i_{f}}\right)_{j},\left(e_{x_{f}}\right)_{y}\right)=0 \Leftrightarrow\left(e_{i_{f}}\right)_{j}=\left(e_{x_{f}}\right)_{y} \\
\tilde{d}\left(\left(e_{i_{f}}\right)_{j},\left(e_{x_{f}}\right)_{y}\right)=\tilde{d}\left(\left(e_{x_{f}}\right)_{y},\left(e_{i_{f}}\right)_{j}\right) \\
\tilde{d}\left(\left(e_{i_{f}}\right)_{j},\left(e_{x_{f}}\right)_{y}\right) \leq \tilde{d}\left(\left(e_{i_{f}}\right)_{j},\left(e_{s_{f}}\right)_{k},\left(e_{x_{f}}\right)_{y}\right)
\end{gathered}
$$

If $\tilde{\mathrm{d}}$ is a soft metric on the soft set $\mathrm{f}$ then, $\mathrm{f}$ is called soft metric space and denoted by (f, $\tilde{\mathrm{d}})$.

Definition 3.2. [11] Let $\left(\mathrm{e}_{\mathrm{i}_{\mathrm{f}}}\right)_{\mathrm{j}}$ and $\left(\mathrm{e}_{\mathrm{x}_{\mathrm{f}}}\right)_{\mathrm{y}}$ be soft points of a soft metric space. The value of $\tilde{d}\left(\left(e_{i_{f}}\right)_{j},\left(e_{x_{f}}\right)_{y}\right)$ is called as the soft distance between the soft points $\left(e_{i_{f}}\right)_{j}$ and $\left(e_{x_{f}}\right)_{y}$.

Definition 3.3. [4] Let (f, $\tilde{\mathrm{d}})$ be a soft metric space $\mathrm{g} \neq \Phi$ and $\mathrm{g} \tilde{\mathrm{f}} \mathrm{f}$. Then the diameter of $\mathrm{g}$ is denoted by $\delta(\mathrm{g})$ and is defined by

$$
\mathrm{d}((\mathrm{g})(\gamma))=\sup \left\{\tilde{\mathrm{d}}\left(\left(\mathrm{e}_{\mathrm{i}_{\mathrm{f}}}\right)_{\mathrm{j}},\left(\mathrm{e}_{\mathrm{x}_{\mathrm{f}}}\right)_{\mathrm{y}}\right) ;\left(\mathrm{e}_{\mathrm{i}_{\mathrm{f}}}\right)_{\mathrm{j}},\left(\mathrm{e}_{\mathrm{x}_{\mathrm{f}}}\right)_{\mathrm{y}} \tilde{\in} \mathrm{g}\right\}, \forall \gamma \tilde{\in} \mathrm{f}
$$

Definition 3.4. [4] Let (f, $\tilde{\mathrm{d}})$ be a soft metric space, $\left(\mathrm{e}_{\mathrm{x}_{\mathrm{f}}}\right)_{\mathrm{y}}$ be a fixed soft point of $\mathrm{f}$ and $\mathrm{g} \tilde{\subseteq} \mathrm{f}$. Then the distance of the soft point $\left(e_{x_{f}}\right)_{y}$ from the soft set $g$ is denoted by $\delta\left(\left(\mathrm{e}_{\mathrm{x}_{\mathrm{f}}}\right)_{\mathrm{y}}, \mathrm{g}\right)$ and defined by

$$
\delta\left(\left(\mathrm{e}_{\mathrm{x}_{\mathrm{f}}}\right)_{\mathrm{y}}, \mathrm{g}\right)(\gamma)=\inf \left\{\tilde{\mathrm{d}}\left(\left(\mathrm{e}_{\mathrm{x}_{\mathrm{f}}}\right)_{\mathrm{y}},\left(\mathrm{e}_{\mathrm{i}_{\mathrm{f}}}\right)_{\mathrm{j}}\right)(\gamma) ;\left(\mathrm{e}_{\mathrm{i}_{\mathrm{f}}}\right)_{\mathrm{j}} \tilde{\epsilon} \mathrm{g}\right\}
$$

Definition 3.5. [4] Let (f, $\tilde{\mathrm{d}})$ a soft metric space, $\tilde{\mathrm{r}}$ be a non-negative soft real number and $\left(\left(\mathrm{e}_{\mathrm{i}_{\mathrm{f}}}\right)_{\mathrm{j}},\left(\mathrm{e}_{\mathrm{x}_{\mathrm{f}}}\right)_{\mathrm{y}}\right) \tilde{\epsilon} \mathrm{f}$.

$$
\begin{aligned}
& \tilde{\mathrm{B}}_{\tilde{\mathrm{d}}}\left(\left(\mathrm{e}_{\mathrm{x}_{\mathrm{f}}}\right)_{\mathrm{y}}, \tilde{\mathrm{r}}\right)=\left\{\left(\mathrm{e}_{\mathrm{i}_{\mathrm{f}}}\right)_{\mathrm{j}} \tilde{\in} \mathrm{f}: \tilde{\mathrm{d}}\left(\left(\mathrm{e}_{\mathrm{i}_{\mathrm{f}}}\right)_{\mathrm{j}},\left(\mathrm{e}_{\left.\left.\left.\mathrm{x}_{\mathrm{f}}\right)_{\mathrm{y}}\right) \tilde{<} \tilde{\mathrm{r}}\right\}}\right.\right.\right. \\
& \tilde{\mathrm{B}}_{\tilde{\mathrm{d}}}\left(\left(\mathrm{e}_{\mathrm{x}_{\mathrm{f}}}\right)_{\mathrm{y}}, \tilde{\mathrm{r}}\right)=\left\{\left(\mathrm{e}_{\mathrm{i}_{\mathrm{f}}}\right)_{\mathrm{j}} \tilde{\in} \mathrm{f}: \tilde{\mathrm{d}}\left(\left(\mathrm{e}_{\mathrm{i}_{\mathrm{f}}}\right)_{\mathrm{j}},\left(\mathrm{e}_{\mathrm{x}_{\mathrm{f}}}\right)_{\mathrm{y}}\right) \tilde{\leq} \tilde{\mathrm{r}}\right\}
\end{aligned}
$$

$$
\tilde{\mathrm{K}}_{\tilde{\mathrm{d}}}\left(\left(\mathrm{e}_{\mathrm{x}_{\mathrm{f}}}\right)_{\mathrm{y}}, \tilde{\mathrm{r}}\right)=\left\{\left(\mathrm{e}_{\mathrm{i}_{\mathrm{f}}}\right)_{\mathrm{j}} \tilde{\epsilon} f: \tilde{\mathrm{d}}\left(\left(\mathrm{e}_{\mathrm{i}_{\mathrm{f}}}\right)_{\mathrm{j}},\left(\mathrm{e}_{\mathrm{x}_{\mathrm{f}}}\right)_{\mathrm{y}}\right) \tilde{=} \tilde{\mathrm{r}}\right\}
$$

These soft sets are called respectively soft open sphere, soft closed sphere and soft k-sphere with center $\left(\mathrm{e}_{\mathrm{x}_{\mathrm{f}}}\right)_{\mathrm{y}}$ and $\operatorname{radius} \tilde{\mathrm{r}}$.

For the sake of clarity, the notation can be written as $\tilde{\mathrm{B}}_{\tilde{\mathrm{d}}}\left(\left(\mathrm{e}_{\mathrm{x}_{\mathrm{f}}}\right)_{\mathrm{y}}, \tilde{\mathrm{r}}\right) \tilde{=} \tilde{\mathrm{B}}\left(\left(\mathrm{e}_{\mathrm{x}_{\mathrm{f}}}\right)_{\mathrm{y}}, \tilde{\mathrm{r}}\right)$.

Besides, there is a property of soft spheres that is valid for all soft metrics:

For $\tilde{\mathrm{r}}_{1}<\tilde{\mathrm{r}}_{2}$,

$$
\tilde{\mathrm{B}}\left(\left(\mathrm{e}_{\mathrm{x}_{\mathrm{f}}}\right)_{\mathrm{y}}, \tilde{\mathrm{r}}_{1}\right) \tilde{\subset} \tilde{\mathrm{B}}\left(\left(\mathrm{e}_{\mathrm{x}_{\mathrm{f}}}\right)_{\mathrm{y}}, \tilde{\mathrm{r}}_{1}\right) \tilde{\subset} \tilde{\mathrm{B}}\left(\left(\mathrm{e}_{\mathrm{x}_{\mathrm{f}}}\right)_{\mathrm{y}}, \tilde{\mathrm{r}}_{2}\right) \tilde{\subset} \tilde{\mathrm{B}}\left(\left(\mathrm{e}_{\mathrm{x}_{\mathrm{f}}}\right)_{\mathrm{y}}, \tilde{\mathrm{r}}_{2}\right)
$$

It is obvious from the definition of soft metric.

After this definition, it is natural to try to relate soft spheres to soft real numbers and soft points. The construction of spheres can be made with soft real numbers and soft points can be seen in the examples:

Example 3.6. Let $\mathrm{d}_{1}$ function is defined as $\tilde{d}_{1}\left(\left(e_{i_{f}}\right)_{j},\left(e_{x_{f}}\right)_{y}\right)=\sqrt{(\bar{i}+\bar{x})^{2}+(\bar{j}+\bar{y})^{2}}$ in $\mathrm{f}$. Then, show the following cases:

(1) (f, $\tilde{\mathrm{d}})$ is a soft metric space.

(2) Find the soft sets $\tilde{B}_{\tilde{d}_{1}}$.

Solution:

$$
\tilde{\mathrm{d}}_{1}: \mathrm{E} ? \mathrm{E} \rightarrow \mathrm{R}^{*}(\mathrm{E})
$$

$$
\left(\left(e_{i_{f}}\right)_{j},\left(e_{x_{f}}\right)_{y}\right) \rightarrow \tilde{d}_{1}\left(\left(e_{i_{f}}\right)_{j},\left(e_{x_{f}}\right)_{y}\right)=\sqrt{(\bar{i}+\bar{x})^{2}+(\bar{j}+\bar{y})^{2}}
$$

$\left(m_{1}\right)$ The square root function is positive defined. Hence, this function is positive, too.

$$
\begin{gathered}
\left(\mathrm{m}_{2}\right) \tilde{d}_{1}\left(\left(\mathrm{e}_{\mathrm{i}_{\mathrm{f}}}\right)_{\mathrm{j}},\left(\mathrm{e}_{\mathrm{x}_{\mathrm{f}}}\right)_{\mathrm{y}}\right)=\overline{0} \Leftrightarrow\left(\mathrm{e}_{\mathrm{i}_{\mathrm{f}}}\right)_{\mathrm{j}}=\left(\mathrm{e}_{\mathrm{x}_{\mathrm{f}}}\right)_{\mathrm{y}}(?) \\
\sqrt{(\overline{\mathrm{i}}+\overline{\mathrm{x}})^{2}+(\overline{\mathrm{j}}+\overline{\mathrm{y}})^{2}}=\overline{0} \Rightarrow(\overline{\mathrm{i}}+\overline{\mathrm{x}})^{2}+(\overline{\mathrm{j}}+\overline{\mathrm{y}})^{2}=\overline{0} \text {. Then, } \\
(\overline{\mathrm{i}}+\overline{\mathrm{x}})^{2}=\overline{0} \text { and }(\overline{\mathrm{j}}+\overline{\mathrm{y}})^{2}=\overline{0} \\
\left(\mathrm{~m}_{3}\right)_{\mathrm{d}_{1}}\left(\left(\mathrm{e}_{\mathrm{i}_{\mathrm{f}}}\right)_{\mathrm{j}},\left(\mathrm{e}_{\mathrm{x}_{\mathrm{f}}}\right)_{\mathrm{y}}\right)=\tilde{\mathrm{d}}_{1}\left(\left(\mathrm{e}_{\mathrm{x}_{\mathrm{f}}}\right)_{\mathrm{y}}\left(\mathrm{e}_{\mathrm{i}_{\mathrm{f}}}\right)_{\mathrm{j}}\right)(?) \\
\text { If square function is applied both of sides, it is obvious. } \\
\left(\mathrm{m}_{4}\right) \forall\left(\mathrm{e}_{\mathrm{i}_{\mathrm{f}}}\right)_{\mathrm{j}},\left(\mathrm{e}_{\mathrm{x}_{\mathrm{f}}}\right)_{\mathrm{y}},\left(\mathrm{e}_{\mathrm{s}_{\mathrm{f}}}\right)_{\mathrm{k}} \tilde{\in} \mathrm{f} . \text { Then, } \\
\mathrm{d}_{1}\left(\left(\mathrm{e}_{\mathrm{i}_{\mathrm{f}}}\right)_{\mathrm{j}},\left(\mathrm{e}_{\mathrm{x}_{\mathrm{f}}}\right)_{\mathrm{y}}\right) \leq \mathrm{d}_{1}\left(\left(\mathrm{e}_{\mathrm{i}_{\mathrm{f}}}\right)_{\mathrm{j}},\left(\mathrm{e}_{\mathrm{s}_{\mathrm{f}}}\right)_{\mathrm{k}}\right)+\tilde{\mathrm{d}}_{1}\left(\left(\left(\mathrm{e}_{\mathrm{s}_{\mathrm{f}}}\right)_{\mathrm{k}}\right),\left(\left(\mathrm{e}_{\mathrm{x}_{\mathrm{f}}}\right)_{\mathrm{y}}\right)\right)
\end{gathered}
$$




$$
\begin{aligned}
\tilde{B}_{1}((\overline{0}, \overline{0}), \overline{2}) & =\left\{\left(e_{\mathrm{i}_{\mathrm{f}}}\right)_{\mathrm{j}} \tilde{\epsilon} \mathrm{f} \mid \tilde{\mathrm{d}}_{1}((\overline{0}, \overline{0}),(\overline{\mathrm{i}}, \overline{\mathrm{j}})) \tilde{<} \overline{2}\right\} \\
& =\left\{\left(\mathrm{e}_{\mathrm{i}_{\mathrm{f}}}\right)_{\mathrm{j}} \tilde{\epsilon} \mathrm{f} \mid \sqrt{(\overline{\mathrm{i}}+\overline{\mathrm{x}})^{2}+(\overline{\mathrm{j}}+\overline{\mathrm{y}})^{2}} \tilde{<} \overline{2}\right\} \\
& =\left\{\left(\mathrm{e}_{\mathrm{i}_{\mathrm{f}}}\right)_{\mathrm{j}} \tilde{\epsilon} \mathrm{f} \mid \mathrm{i}^{2}+\mathrm{j}^{2} \tilde{<} \overline{4}\right\}, \tilde{\mathrm{r}}=\overline{2} \text { and } O(\overline{0}, \overline{0})
\end{aligned}
$$

Remark 3.7. Any soft intersection of soft open spheres need not to be soft open. The following example shows that: Example 3.8. Let me show the equation

$$
\begin{aligned}
& \bigcap_{\bar{n}=1}^{\infty} \tilde{\mathrm{B}}\left(\left(\mathrm{e}_{\mathrm{x}_{\mathrm{f}}}\right)_{\mathrm{y}}, \overline{1}+\frac{\overline{1}}{\overline{\mathrm{n}}}\right)=\tilde{\mathrm{B}}\left(\left(\mathrm{e}_{\mathrm{x}_{\mathrm{f}}}\right)_{\mathrm{y}}, \overline{1}\right) \\
& \text { a) } \bigcap_{\overline{\mathrm{n}}=1}^{\infty} \tilde{\mathrm{B}}\left(\left(\mathrm{e}_{\mathrm{x}_{\mathrm{f}}}\right)_{\mathrm{y}}, \overline{1}+\frac{\overline{1}}{\overline{\mathrm{n}}}\right) \tilde{\subset} \tilde{\mathrm{B}}\left(\left(\mathrm{e}_{\mathrm{x}_{\mathrm{f}}}\right)_{\mathrm{y}}, \overline{1}\right)(?) \\
& \left(\mathrm{e}_{\mathrm{i}_{\mathrm{f}}}\right)_{\mathrm{j}} \tilde{\epsilon} \bigcap_{\overline{\mathrm{n}}=1}^{\infty} \tilde{\mathrm{B}}\left(\left(\mathrm{e}_{\mathrm{x}_{\mathrm{f}}}\right)_{\mathrm{y}}, \overline{1}+\frac{\overline{1}}{\overline{\bar{n}}}\right) \Rightarrow\left(\mathrm{e}_{\mathrm{i}_{\mathrm{f}}}\right)_{\mathrm{j}} \tilde{\in} \tilde{\mathrm{B}}\left(\left(\mathrm{e}_{\mathrm{x}_{\mathrm{f}}}\right)_{\mathrm{y}}, \overline{1}+\frac{\overline{1}}{\overline{\mathrm{n}}}\right) \Rightarrow|\overline{\mathrm{i}}-\overline{\mathrm{x}}| \tilde{<} \overline{1}+\frac{\overline{1}}{\overline{\mathrm{n}}} \\
& \left.\begin{array}{l}
(\forall \mathcal{E}>0), \overline{\mathrm{i}} \tilde{<} \overline{\mathrm{j}}+\varepsilon, \overline{\mathrm{i}} \tilde{\geq} \overline{\mathrm{j}} \\
(\forall \varepsilon>0)\left(\frac{\overline{1}}{\overline{\mathrm{n}}} \tilde{<} \varepsilon\right): \exists \overline{\mathrm{n}} \tilde{\in} \mathbb{N}
\end{array}\right\} \Rightarrow|\overline{\mathrm{i}}-\overline{\mathrm{x}}| \tilde{<} \overline{1}+\frac{\overline{1}}{\overline{\mathrm{n}}} \tilde{\leq} \overline{1} \Rightarrow\left(\mathrm{e}_{\mathrm{i}_{\mathrm{f}}}\right)_{\mathrm{j}} \tilde{\in} \tilde{\mathrm{B}}\left(\left(\mathrm{e}_{\mathrm{x}_{\mathrm{f}}}\right)_{\mathrm{y}}, \overline{1}\right) \\
& \text { b) }\left(\mathrm{e}_{\mathrm{i}_{\mathrm{f}}}\right)_{\mathrm{j}} \tilde{\epsilon} \tilde{\mathrm{B}}\left(\left(\mathrm{e}_{\mathrm{x}_{\mathrm{f}}}\right)_{\mathrm{y}}, \overline{1}\right) \Rightarrow \tilde{\rho}\left(\left(\mathrm{e}_{\mathrm{i}_{\mathrm{f}}}\right)_{\mathrm{j}},\left(\mathrm{e}_{\mathrm{x}_{\mathrm{f}}}\right)_{\mathrm{y}}\right) \tilde{\leq} \overline{1}<\overline{1}+\overline{1} \overline{\bar{n}},\left(\exists \overline{\mathrm{n}} \tilde{\in} \mathbb{N}^{+}\right) \\
& \tilde{\rho}\left(\left(\mathrm{e}_{\mathrm{i}_{\mathrm{f}}}\right)_{\mathrm{j}},\left(\mathrm{e}_{\mathrm{x}_{\mathrm{f}}}\right)_{\mathrm{y}}\right) \tilde{\leq} \overline{1}+\frac{\overline{1}}{\overline{\mathrm{n}}} \Rightarrow\left(\mathrm{e}_{\mathrm{i}_{\mathrm{f}}}\right)_{\mathrm{j}} \tilde{\epsilon} \bigcap_{\overline{\mathrm{n}}=1}^{\infty} \tilde{\mathrm{B}}\left(\left(\mathrm{e}_{\mathrm{x}_{\mathrm{f}}}\right)_{\mathrm{y}}, \overline{1}+\frac{\overline{1}}{\overline{\mathrm{n}}}\right)
\end{aligned}
$$

Remark 3.9. Any soft union of soft closed spheres need not to be soft closed. The following example shows that:

Example 3.10. Let me show the equation $\bigcup_{n=1}^{\infty} \tilde{\mathrm{B}}\left(\left(\mathrm{e}_{\mathrm{x}_{\mathrm{f}}}\right)_{\mathrm{y}}, \frac{\overline{\mathrm{n}}}{\overline{\mathrm{n}}+\overline{1}}\right) \approx B\left(\left(\mathrm{e}_{\mathrm{x}_{\mathrm{f}}}\right)_{\mathrm{y}}, \overline{1}\right)$.

$$
\begin{gathered}
\mathrm{a}) \bigcup_{n=1}^{\infty} \tilde{\mathrm{B}}\left(\left(\mathrm{e}_{\mathrm{x}_{\mathrm{f}}}\right)_{\mathrm{y}}, \frac{\overline{\mathrm{n}}}{\overline{\mathrm{n}}+\overline{1}}\right) \tilde{\subset} \mathrm{B}\left(\left(\mathrm{e}_{\mathrm{x}_{\mathrm{f}}}\right)_{\mathrm{y}}, \overline{1}\right)(?) \\
\exists\left(\mathrm{e}_{\mathrm{x}_{\mathrm{f}}}\right)_{\mathrm{y}} \tilde{\epsilon} \tilde{B}\left(\left(\mathrm{e}_{\mathrm{x}_{\mathrm{f}}}\right)_{\mathrm{y}}, \frac{\tilde{n}}{n+1}\right) \Rightarrow \tilde{\rho}\left(\left(\mathrm{e}_{\mathrm{x}_{\mathrm{f}}}\right)_{\mathrm{y}},\left(\mathrm{e}_{i_{\mathrm{f}}}\right)_{j}\right) \tilde{\leq} \frac{\tilde{n}}{n+1} \tilde{<} \overline{1}\left(\forall \bar{n} \tilde{\epsilon} \mathbb{N}^{+}\right) \\
\Rightarrow \tilde{\rho}\left(\left(\mathrm{e}_{\mathrm{x}_{\mathrm{f}}}\right)_{\mathrm{y}},\left(\mathrm{e}_{\mathrm{i}_{\mathrm{f}}}\right)_{j}\right) \tilde{<} \overline{1} \Rightarrow\left(\mathrm{e}_{\mathrm{i}_{\mathrm{f}}}\right)_{j} \tilde{\epsilon} B_{\rho}\left(\left(\mathrm{e}_{\mathrm{x}_{\mathrm{f}}}\right)_{\mathrm{y}}, \overline{1}\right) \\
\mathrm{b})\left(\mathrm{e}_{i_{\mathrm{f}}}\right)_{j} \tilde{\epsilon} \tilde{B}\left(\left(\mathrm{e}_{\mathrm{x}_{\mathrm{f}}}\right)_{\mathrm{y}}, \overline{1}\right) \Rightarrow\left(\mathrm{e}_{\mathrm{i}_{\mathrm{f}}}\right)_{\mathrm{j}} \tilde{\epsilon} \bigcup_{\bar{n}=\overline{1}}^{\infty} \tilde{B}\left(\left(\mathrm{e}_{\mathrm{x}_{\mathrm{f}}}\right)_{\mathrm{y}}, \frac{\bar{n}}{\bar{n}+1}\right)(?) \\
\left(\mathrm{e}_{\mathrm{i}_{\mathrm{f}}}\right)_{j} \tilde{\epsilon} \tilde{B}\left(\left(\mathrm{e}_{\mathrm{x}_{\mathrm{f}}}\right)_{\mathrm{y}}, \overline{1}\right) \Rightarrow \tilde{\rho}\left(\left(\mathrm{e}_{\mathrm{x}_{\mathrm{f}}}\right)_{\mathrm{y}},\left(\mathrm{e}_{\mathrm{i}_{\mathrm{f}}}\right)_{\mathrm{j}}\right) \tilde{<} \overline{1}
\end{gathered}
$$

Suppose that, $\left(\mathrm{e}_{\mathrm{x}_{\mathrm{f}}}\right)_{\mathrm{y}}<\left(\mathrm{e}_{\mathrm{i}_{\mathrm{f}}}\right)_{\mathrm{j}}<\left(\mathrm{e}_{\mathrm{x}_{\mathrm{f}}}\right)_{\mathrm{y}}+\frac{\overline{1}}{\bar{n}}$ and $(1 / \bar{n}=\varepsilon)$. This leads to the contradiction that $x \in B(a, 1)$. Hence, Then $\left(e_{x_{f}}\right)_{y}<\left(e_{i_{f}}\right)_{j} \leq\left(e_{x_{f}}\right)_{y}$. So, $\left(e_{x_{f}}\right)_{y}=\left(e_{i_{f}}\right)_{j}$. 


$$
\begin{aligned}
& \left(\mathrm{e}_{\mathrm{x}_{\mathrm{f}}}\right)_{\mathrm{y}}+\frac{\overline{1}}{\bar{n}}<\left(\mathrm{e}_{\mathrm{i}_{\mathrm{f}}}\right)_{\mathrm{j}} \\
& \Rightarrow\left(\mathrm{e}_{\mathrm{x}_{\mathrm{f}}}\right)_{\mathrm{y}}<\frac{\bar{n}}{a n+1} \leq \frac{\bar{n}}{n+1} \\
& \Rightarrow\left(\mathrm{e}_{\mathrm{x}_{\mathrm{f}}}\right)_{\mathrm{y}}<\left(\mathrm{e}_{\mathrm{i}_{\mathrm{f}}}\right)_{\mathrm{j}} \leq \frac{\bar{n}}{n+1} \\
& \Rightarrow\left(\mathrm{e}_{\mathrm{i}_{\mathrm{f}}}\right)_{\mathrm{j}}<\left(\left(\mathrm{e}_{\mathrm{x}_{\mathrm{f}}}\right)_{\mathrm{y}}, \frac{\bar{n}}{n+1}\right) \\
& \Rightarrow \tilde{\rho}\left(\left(\mathrm{e}_{\mathrm{x}_{\mathrm{f}}}\right)_{\mathrm{y}},\left(\mathrm{e}_{\mathrm{i}_{\mathrm{f}}}\right)_{\mathrm{j}}\right) \tilde{\leq} \frac{\bar{n}}{n+1}
\end{aligned}
$$

\section{Conclusion}

This study is intended as an attempt to motivate bringing together the areas in which soft spheres, soft real numbers and soft points. Relating spheres to soft real numbers and soft points provides a natural and intrinsic construction of soft spheres. This viewpoint sheds some new light on soft sphere examples and drawings for analyzing the locus of them.

\section{Results and Discussions}

The notions defined in A. Dress, K. T. Huber, V. Moulton (2001) and S. Das and S.K. Samanta (2012), it is natural to think that there is a close relation between the characterization problem of spheres and the characterization problem of soft real numbers. In this paper, a construction of spheres is provided with soft real numbers and soft points, so the above problem can be considered for further research in case of illustrate spheres in soft metric theory. As a further study, the construction of these spheres represented by using soft real numbers may be drawn. This new approach may be the starting point for soft mathematical concepts and structures based on soft set-theoric operations in soft metric spaces and stimulate the reader to further research.

\section{References}

[1] D. Molodtsov, Soft set theory-first results, Comput. Math. Appl.(37) (1999) 19-31.

[2] M.I. Ali, F. Feng, X. Liu, W.K. Min and M. Shabir, On some new operations in soft set theory, Comput. Math. Appl. 57 (2009), 1547-1553.

[3] N. Cagman and S. Enginoglu, Soft matrix theory and its decision making, Comput. Math. Appl.(59)(2010), 3308-3314.

[4] S. Das and S.K. Samanta, Soft Metric Spaces, Annals of Fuzzy Mathematics and Informatics, 6(1) (2013) 77-94.

[5] G. Senel, The Parameterization Reduction of Soft Point and its Applications with Soft Matrix, International Journal of Computer Applications, (164) (1) (2017), 1-6.

[6] S. Das and S.K. Samanta, Soft Real Sets, Soft Real Numbers and Their Properties, J. Fuzzy Math. 20 (3) (2012) 551-576.

[7] S. Das and S.K. Samanta, On soft complex sets and soft complex numbers, The Journal of Fuzzy Mathematics (21)(1) (2013) 195-216.

[8] A. Dress, K. T. Huber, V. Moulton, Metric Spaces in Pure and Applied Mathematics, Documenta Mathematica Quadratic Forms LSU (2001), 121-139.

[9] S. Semmes, Some Remarks About Metric Spaces, Spherical Mappings, Functions and Their Derivatives, Publicacions Matem'atiques 40(1996), 411-430.

[10] A. Aygunoğlu, H. Aygun, Some notes on soft topological spaces, Neural Comp. Appl. (2011), 521-011-0722-3.

[11] G. Senel, Soft Metric Spaces, Gaziosmanpasa University Graduate School of Natural and Applied Sciences Department of Mathematics Ph.D. Thesis, (2013), 92. 